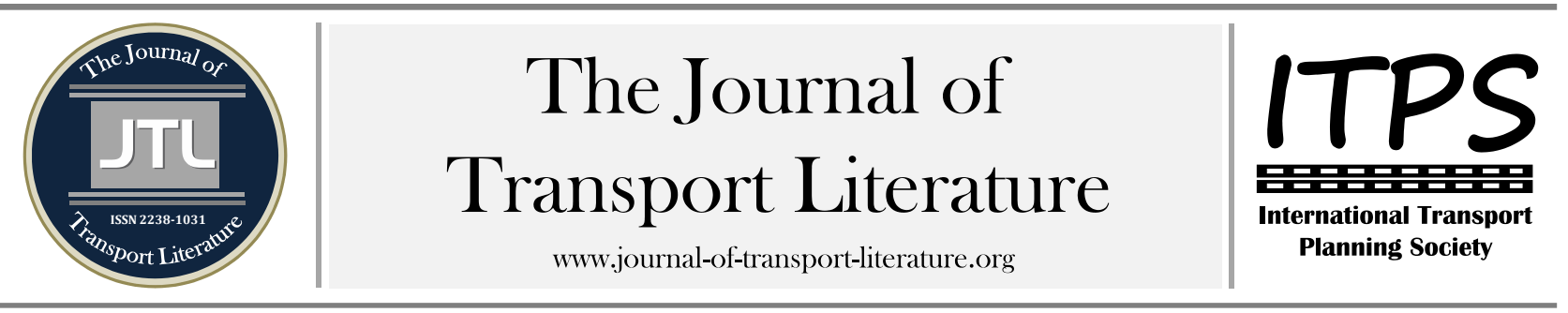

\title{
Análise de desempenho operacional em terminais de contêineres brasileiros
}

\author{
Ana Maria Volkmer de Azambuja ${ }^{1,+} ;$ Maiquiel Schmidt de Oliveira ${ }^{2}$; Milton Luiz Paiva de Lima ${ }^{1}$ \\ ${ }_{1}^{1}$ Universidade Federal do Rio Grande - FURG, Rio Grande, Brasil \\ ${ }^{2}$ Universidade Tecnológica Federal do Paraná - UTFPR, Francisco Beltrão, Brasil
}

\section{Article Info}

\section{Resumo}

Palavras-chave:

Terminais de Contêineres

Desempenho Operacional

Eficiência Técnica

Análise de Janelas

Submitted 27 Jun 2014

27 Feb 2015

accepted 10 May 2015.

Licensed under

Creative Common

CC-BY $3.0 \mathrm{BR}$

\begin{abstract}
Com o grande crescimento nas últimas décadas da movimentação de cargas por contêineres, os terminais de contêineres vem contribuindo, de forma significativa, na cadeia logística nacional e internacional. Como forma de aumentarem sua produtividade, esses terminais precisam conhecer seu potencial de captação de mercadorias e suas limitações. Esse estudo teve como objetivo observar as tendências de crescimento ou redução na produtividade de terminais de contêineres brasileiros, ao longo de determinado período de tempo. 0 período analisado foi de 2004 a 2011. Para tal, utilizou-se um modelo DEA conhecido como Modelo de Janelas. Para Análise de Janelas foi utilizado escore estimado a partir do modelo BCC com orientação para produto. Para o período analisado, o terminal TECON Santos foi o único que se manteve eficiente ao longo de todo o período. Os terminais CONVICON (PA), Libra-Rio, Libra Santos (SP) e Tecon Rio Grande (RS) se mostraram eficientes na movimentação de contêineres em parte desse período.
\end{abstract}

+ Corresponding author. Universidade Federal do Rio Grande, Escola de Engenharia. Av. Itália, km 8. CEP 96.203-000 - Rio Grande, RS - Brasil. E-mail address: anaazambuja@furg.br.

\section{Introdução}

Com o grande crescimento nas últimas décadas da movimentação de cargas por contêineres, os terminais de contêineres vem contribuindo, de forma significativa, na cadeia logística tanto à nível nacional como internacional. Como forma de aumentarem sua produtividade, esses terminais precisam se especializar, conhecendo seu potencial de captação de mercadorias e suas limitações.

Esse estudo teve como objetivo observar as tendências de crescimento ou redução na produtividade de terminais de contêineres brasileiros, ao longo de determinado período de tempo. O período analisado foi de 2004 a 2011 . Para tal, utilizou um modelo DEA conhecido como Modelo de Janelas. Esse modelo parte de um escore de eficiência, que foi estimado para o ano de 2011 em estudo anterior (Oliveira, 2012). O modelo utilizado foi o BCC com orientação para produto. A escolha desse modelo se deu a partir da verificação que os terminais de contêineres brasileiros variam em porte. A orientação para produto foi escolhida porque quer se utilizar toda a infraestrutura existente para aumentar a produtividade quando possível.

O Modelo de janelas permite que se analise as variações na eficiência técnica relativa de determinada unidade ao longo do tempo.

0 presente artigo, além da introdução, apresenta a Seção 1 com a metodologia utilizada para a análise dos terminais de contêineres, onde se conceitua o Modelo de Janelas. Na seção 2 se faz aplicação do modelo considerando dados referente ao período de 2004 a 2011. Na seção 3 é feita uma caracterização dos terminais que se mostraram eficientes em pelo menos parte desse período. Por fim, são apresentadas as principais conclusões.

\section{Metodologia}

A técnica conhecida como Análise Envoltória de Dados (DEA) vem sendo amplamente aplicada em diversas áreas do conhecimento. Esta técnica possibilita, a partir de modelo apropriado, comparar unidades semelhantes em relação ao seu desempenho. Porém, essa análise se torna mais completa quando verificada a evolução dessas unidades ao longo do tempo. Assim, aplica-se nesse estudo o modelo DEA de Análise de Janelas, que possibilita verificar o desempenho desses terminais de contêineres em determinado período.

\subsection{Modelo DEA de Análise de Janelas}

Os modelos DEA podem trabalhar com retornos de escala constantes (modelo CCR - Charles, Cooper e Rhodes, também conhecido como CRS) ou com retornos de escala variáveis (modelo BCC - Banker, Charnes e Cooper, também conhecido como VRS). Além dos retornos de escala, deve-se definir se as unidades ineficientes devem ser projetadas na fronteira de eficiência a partir de aumento da produção (modelo de orientação para produto), redução na quantidade dos insumos 
(modelo de orientação para insumo) ou ambos (modelo aditivo). A partir dessa definição e de dados em um determinado momento, define-se o escore de eficiência, capaz de medir a eficiência relativa dos terminais incluídos na análise. Com a definição desse escore de eficiência, pode-se aplicar o modelo de Análise de Janelas.

Ferreira e Gomes (2009) comentam que os primórdios da Análise de Janela vem de Charnes et al. (1985) visando analisar as variações na eficiência técnica relativa de determinada unidade ao longo do tempo. Essa abordagem permite analisar o desempenho temporal das unidades em análise, oferecendo evidências de estabilidade e sensibilidade a partir dos escores de eficiência técnica de cada unidade. 0 modelo de Análise de Janelas utiliza uma abordagem semelhante à Análise Estatística Móvel, isto é, cada vez que se inclui um novo período de tempo na análise, o anterior é retirado.

Cullinane et al. (2004) afirmam que existem vários estudos avaliando eficiência na produção em terminais de contêineres utilizando DEA em um determinado momento no tempo. Destacam, ainda, que quando o tempo não é considerado, os resultados de eficiência derivados desta metodologia podem ser tendenciosos. Afim de superar este problema, sugerem a aplicação do modelo de Análise de Janelas, utilizando dados em panel. Esses autores aplicaram este modelo para avaliar uma amostra dos 25 principais terminais de contêineres do mundo. Essa análise levou os autores desse artigo a utilizarem método semelhante como forma de complementar avaliação anterior apresentada em Oliveira et. al. (2013) para avaliar terminais de contêineres brasileiros.

Para a aplicação desse modelo é necessário calcular o número de janelas, dado por:

$$
\mathrm{n}=\mathrm{p}-\mathrm{w}+1
$$

onde:

n - número de janelas;

p - número de períodos e

$\mathrm{w}$ - tamanho das janelas.

O número de unidades virtuais é dado por:

$$
\text { № unidades virtuais }=\mathrm{k} \times \mathrm{w} \times \mathrm{n}
$$

onde:

$\mathrm{k}$ - número de unidades.

O número de unidades em cada janela é dado por:

$$
\text { № de unidades por janela }=\mathrm{k} \times \mathrm{w} / 2
$$

O tamanho das janelas utilizadas em uma análise é dado por:

$$
\mathrm{w}=\frac{\mathrm{p}+1}{2}
$$

Lovell (1996) ressalta que a abordagem da Análise de Janelas (Window Analysis) não fornece nenhuma evidência sobre a natureza do progresso tecnológico (deslocamento da fronteira) e mostra pouca informação sobre as mudanças na produtividade. Porém, essa análise auxilia na avaliação temporal de cada unidade, podendo-se supor ou verificar, caso existam, possíveis causas de variações nos escores de eficiência.

Para aplicação do modelo de Análise de Janelas foi utilizado o mesmo escore de eficiência desenvolvido em Oliveira (2012).

Nesse estudo foi utilizado tamanho de janela igual a três, o que é sugerido no trabalho original de Charnes, Clark, Cooper e Golany (1985), precursores dessa análise.

\section{Análise dos Dados}

$\mathrm{Na}$ análise foram considerados 13 terminais de contêineres: Libra Terminais Rio (RJ), Libra Terminais Santos (SP), MultiRio (RJ), TECON Rio Grande (RS), TECON Salvador (BA), TECON Santos (SP), TECON Sepetiba (RJ), TECON Suape (PE). Terminal de Contêineres de Itajaí - TECONVI (SC), Terminal de Contêineres de Paranaguá- TCP (PR), Terminal de Contêineres de Vila do Conde - CONVICON (PA), Terminal de Vila Velha - TVV (ES) e Terminal para Contêineres da Margem Direita - TECONDI(SP).

O escore de eficiência utilizado na análise, desenvolvido na dissertação de Oliveira (2012), foi definido a partir de dados referentes às variáveis:

- Insumos: área total do terminal $\left(\mathrm{m}^{2}\right)$, profundidade do canal de acesso (m), profundidade do cais de atracação (m), número de berços, número de acessos e extensão dos berços (m);

- Produto: movimentação de contêineres (TEU's).

Esses dados se referem ao ano de 2011. 0 escore estimado considerou retornos variáveis à escala e orientação para produto.

$$
\text { Eficiência }=\frac{\text { Movimentação de Contêineres }}{\text { Número de Acessos+Número de Berços }}
$$

Para aplicação do modelo de Análise de Janelas foi utilizado o escore apresentado em (5), para o período de 2004 a 2011. 
A análise foi realizada a partir do ano de 2004 porque o Terminal de Vila do Conde (PA) iniciou suas atividades em novembro de 2003. Cada terminal de contêineres é representado como se fosse uma unidade diferente em cada uma das três datas observadas na parte superior de cada coluna.

A tabela abaixo apresenta os resultados obtidos.

\begin{tabular}{|c|c|c|c|c|c|c|c|c|c|c|}
\hline DMU & 2004 & 2005 & 2006 & 2007 & 2008 & 2009 & 2010 & 2011 & Média & $\begin{array}{c}\text { Média } \\
\text { por } \\
\text { janela }\end{array}$ \\
\hline CONVI- & 0,73 & 1,00 & 1,00 & & & & & & 0,9099 & \\
\hline $\mathrm{CON}$ & & 1,00 & 1,00 & 0,85 & & & & & 0,9513 & \\
\hline \multirow[t]{4}{*}{$(\mathrm{PA})$} & & & 1,00 & 0,85 & 0,81 & & & & 0,8880 & \\
\hline & & & & 0,89 & 0,85 & 1,00 & & & 0,9134 & \\
\hline & & & & & 0,84 & 1,00 & 1,00 & & 0,9421 & \\
\hline & & & & & & 1,00 & 1,00 & 0,85 & 0,9471 & 0,9253 \\
\hline Libra & 0,26 & 1,00 & 0,24 & & & & & & 0,5010 & \\
\hline Rio & & 1,00 & 0,24 & 0,20 & & & & & 0,4814 & \\
\hline \multirow[t]{4}{*}{ (RJ) } & & & 0,87 & 0,74 & 0,78 & & & & 0,7979 & \\
\hline & & & & 0,69 & 0,73 & 1,00 & & & 0,8068 & \\
\hline & & & & & 0,73 & 1,00 & 0,82 & & 0,8504 & \\
\hline & & & & & & 1,00 & 0,82 & 0,87 & 0,8970 & 0,7224 \\
\hline Libra & & 0,71 & 0,66 & 0,73 & & & & & 0,7016 & \\
\hline Santos & & & 0,88 & 1,00 & 0,97 & & & & 0,9436 & \\
\hline \multirow[t]{3}{*}{ (SP) } & & & & 1,00 & 1,00 & 0,97 & & & 0,9839 & \\
\hline & & & & & 1,00 & 0,97 & 0,77 & & 0,9090 & \\
\hline & & & & & & 0,97 & 0,77 & 0,74 & 0,8271 & 0,8559 \\
\hline Multi- & 0,35 & 0,20 & 0,24 & & & & & & 0,2632 & \\
\hline Rio & & 0,25 & 0,24 & 0,25 & & & & & 0,2454 & \\
\hline \multirow[t]{4}{*}{ (RJ) } & & & 0,86 & 0,92 & 1,00 & & & & 0,9247 & \\
\hline & & & & 0,86 & 0,93 & 0,89 & & & 0,8922 & \\
\hline & & & & & 0,93 & 0,89 & 0,83 & & 0,8844 & \\
\hline & & & & & & 0,89 & 0,83 & 0,88 & 0,8669 & 0,6795 \\
\hline TCP & 0,60 & 0,61 & 0,53 & & & & & & 0,5787 & \\
\hline \multirow{5}{*}{ (PR) } & & 0,62 & 0,66 & 0,61 & & & & & 0,6277 & \\
\hline & & & 0,79 & 0,73 & 0,80 & & & & 0,7754 & \\
\hline & & & & 0,71 & 0,77 & 1,00 & & & 0,8245 & \\
\hline & & & & & 0,77 & 1,00 & 0,88 & & 0,8814 & \\
\hline & & & & & & 1,00 & 0,88 & 0,83 & 0,9014 & 0,7648 \\
\hline TECON- & 0,34 & 0,31 & 0,27 & & & & & & 0,3079 & \\
\hline & & 0,32 & 0,33 & 0,33 & & & & & 0,3273 & \\
\hline (SP) & & & 0,40 & 0,40 & 0,46 & & & & 0,4203 & \\
\hline & & & & 0,43 & 0,50 & 0,57 & & & 0,5015 & \\
\hline & & & & & 0,51 & 0,58 & 0,39 & & 0,4920 & \\
\hline & & & & & & 0,66 & 0,41 & 0,42 & 0,4942 & 0,4239 \\
\hline TECON- & 0,70 & 0,55 & 0,49 & & & & & & 0,5807 & \\
\hline & & 0,55 & 0,49 & 0,40 & & & & & 0,4825 & \\
\hline$(\mathrm{SP})$ & & & 1,00 & 0,82 & 0,77 & & & & 0,8633 & \\
\hline & & & & 0,87 & 0,82 & 0,41 & & & 0,6995 & \\
\hline & & & & & 0,82 & 0,41 & 0,61 & & 0,6157 & \\
\hline & & & & & & 0,41 & 0,61 & 0,56 & 0,5287 & 0,6284 \\
\hline TECON & 1,00 & 1,00 & 0,67 & & & & & & 0,8902 & \\
\hline Rio & & 1,00 & 0,83 & 0,76 & & & & & 0,8634 & \\
\hline Grande & & & 1,00 & 0,92 & 0,91 & & & & 0,9438 & \\
\hline (RS) & & & & 1,00 & 0,99 & 0,84 & & & 0,9437 & \\
\hline & & & & & 1,00 & 0,85 & 0,66 & & 0,8348 & \\
\hline & & & & & & 0,88 & 0,69 & 0,60 & 0,7227 & 0,8664 \\
\hline TECON & 0,33 & 0,27 & 0,32 & & & & & & 0,3045 & \\
\hline Salva- & & 0,33 & 0,34 & 0,31 & & & & & 0,3261 & \\
\hline Dor & & & 0,74 & 0,67 & 0,62 & & & & 0,6768 & \\
\hline$(\mathrm{BA})$ & & & & 0,69 & 0,63 & 0,75 & & & 0,6887 & \\
\hline & & & & & 0,63 & 0,76 & 0,65 & & 0,6814 & \\
\hline & & & & & & 0,77 & 0,66 & 0,60 & 0,6773 & 0,5591 \\
\hline TECON & 1,00 & 1,00 & 1,00 & & & & & & 10,000 & \\
\hline Santos & & 1,00 & 1,00 & 1,00 & & & & & 10,000 & \\
\hline (SP) & & & 1,00 & 1,00 & 1,00 & & & & 10,000 & \\
\hline & & & & 1,00 & 1,00 & 1,00 & & & 10,000 & \\
\hline & & & & & 1,00 & 1,00 & 1,00 & & 10,000 & \\
\hline & & & & & & 1,00 & 1,00 & 1,00 & 10,000 & 1,000 \\
\hline TECON & 0,27 & 0,27 & 0,32 & & & & & & 0,2907 & \\
\hline Sepetiba & & 0,31 & 0,35 & 0,22 & & & & & 0,2917 & \\
\hline (RJ) & & & 0,76 & 0,48 & 0,81 & & & & 0,6838 & \\
\hline & & & & 0,49 & 0,83 & 0,73 & & & 0,6809 & \\
\hline & & & & & 0,83 & 0,73 & 0,73 & & 0,7672 & \\
\hline & & & & & & 0,74 & 0,74 & 0,74 & 0,7435 & 0,5763 \\
\hline TECON & 0,27 & 0,27 & 0,25 & & & & & & 0,2607 & \\
\hline Suape & & 0,27 & 0,27 & 0,30 & & & & & 0,2783 & \\
\hline$(\mathrm{PE})$ & & & 0,59 & 0,65 & 0,76 & & & & 0,6649 & \\
\hline & & & & 0,66 & 0,77 & 0,82 & & & 0,7530 & \\
\hline & & & & & 0,78 & 0,83 & 0,85 & & 0,8185 & \\
\hline & & & & & & 0,84 & 0,86 & 1,00 & 0,8995 & 0,6125 \\
\hline TVV & 0,33 & 0,32 & 0,29 & & & & & & 0,3151 & \\
\hline (ES) & & 0,33 & 0,34 & 0,34 & & & & & 0,3339 & \\
\hline & & & 0,53 & 0,52 & 0,49 & & & & 0,5108 & \\
\hline & & & & 0,55 & 0,52 & 0,51 & & & 0,5255 & \\
\hline & & & & & 0,52 & 0,51 & 0,46 & & 0,4988 & \\
\hline & & & & & & 0,55 & 0,49 & 0,50 & 0,5145 & 0,4498 \\
\hline
\end{tabular}


Analisando a tabela 1 constata-se que não há uma regularidade nos escores de eficiência dos terminais ao longo do tempo, exceto para o terminal TECON Santos (SP), que foi o único que se manteve eficiente durante todos os períodos analisados. Os demais terminais oscilam e apresentam eficiências elevadas em alguns períodos e menores em outros.

0 terminal CONVICON (PA) apresentou um bom desempenho nesse período tendo uma pequena queda em sua eficiência nos anos de 2007, 2008 e 2011. Verificando a movimentação de contêineres nesse período, percebe-se uma queda.

Percebe-se que o terminal Libra-Rio melhorou seu desempenho a partir de 2006, alcançando a fronteira de eficiência em 2009. Nos anos de 2010 e 2011, apesar de ter apresentado aumento na movimentação de contêineres, esse aumento não acompanhou o crescimento geral dos demais terminais sofrendo novamente uma queda nos escores de eficiência.

0 terminal Libra-Santos teve melhorias em seu desempenho a partir de 2006, onde se manteve próximo da fronteira de eficiência até 2009. A recuperação na quantidade de contêineres movimentada nesse período contribuiu para um bom desempenho. Em 2009 enfrentou nova queda na movimentação, onde a recuperação nos anos seguintes não acompanhou o crescimento geral.

O Multi-Rio mostrou bons escores de eficiência no período de 2006 a 2008. Desde então sofreu pequena queda em seus escores, o que pode ter ocorrido em função de redução na movimentação de contêineres.

0 terminal TCP-PR também demonstrou melhorias em sua performance a partir de 2006, chegando à eficiência em 2009. A partir desse ano, apesar de aumento na movimentação de contêineres, esse não acompanhou o crescimento geral.

0 terminal TECONDI-SP vem mantendo seus escores de eficiência baixos nesse período. Observa-se que, apesar de aumento na movimentação de contêineres, esse não acompanhou o crescimento geral. Além disso, em 2010 entrou em operação mais um berço.

0 terminal TECONVI-SP tem apresentado escores de eficiência baixos. Nesse período sofreu queda na sua movimentação de contêineres, onde em 2010 começou pequena recuperação.

O terminal TECON Rio Grande também teve seu pico no período de 2006 e 2008, tendo redução gradativa em sua eficiência a partir desse ano. Percebe-se que, nesse período, manteve a movimentação de contêineres, não acompanhando o crescimento geral. Além disso, em 2009, entrou em operação mais um berço.

O terminal TECON Salvador teve uma boa melhora no seu escore de eficiência a partir de 2005, mantendo-se em torno de $68 \%$. A razão dessa estabilidade pode ser justificada por esse terminal não ter acompanhado o crescimento geral.

O TECON Santos se manteve eficiente em todo o período analisado. Apesar de pequena queda na movimentação de contêineres sofrida em 2009 esse terminal se manteve na fronteira de eficiência.

O terminal TECON Sepetiba (RJ) apresentou uma melhora em seu índice de eficiência a partir de 2006, mantendo-se, desde então, em torno de 72\%. Percebe-se queda na movimentação de contêineres em 2007, recuperando-se nos anos seguintes.

O terminal SUAPE (PE) também apresentou melhorias no seu escore de eficiência a partir de 2006 mantendo escore de aproximadamente $78 \%$.

0 terminal TVV (ES) também apresentou pequena melhora em seu escore de eficiência a partir de 2006, ficando em torno de 51\%. Percebe-se queda significativa na movimentação de contêineres em 2009; vem se recuperando a partir de então.

O número de acessos não se alterou nesse período para nenhum terminal analisado. 0 no de berços aumentou para os terminais TECONDI-SP e TECON Rio Grande-RS.

\section{Caracterização dos Terminais Eficientes}

A seguir serão destacadas as principais características dos terminais que se mostraram eficientes em algum momento do período analisado pelo Modelo de Janelas como forma de entender suas posições no ranking de desempenho operacional.

O terminal CONVICON (PA) apresenta eficiência nas suas operações apesar de movimentar poucas TEU's. Isso devido a ótimo aproveitamento de seus insumos no processo de produção. Dispõe de uma área total de $103 \mathrm{mil} \mathrm{m}^{2}, \mathrm{com}_{7,5} \mathrm{mil} \mathrm{m} \mathrm{m}^{2}$ de armazéns cobertos. Atualmente é administrado pela Santos Brasil S/A, mesma detentora dos direitos do TECON Santos.

0 terminal Libra Rio (RJ) mostrou-se eficiente nos anos de 2005 e 2009, sendo que em ambos os períodos apresentou uma maior contribuição percentual na movimentação de contêineres. Possui uma área de armazenagem de 136 mil m², sendo 9,6 mil de armazéns cobertos. É administrado pela Libra Terminais, a mesma que administra o terminal Libra Santos.

0 terminal Libra Santos (SP) obteve a segunda maior movimentação em TEU's para o período em análise. Esse terminal usufrui de boa parte dos acessos disponíveis para o TECON Santos, pois se localiza próximo. Dispõe de 155 mil $\mathrm{m}^{2}$ de área total, com $11 \mathrm{mil} \mathrm{m}^{2}$ de armazém coberto e uma capacidade estática de 15,6 mil TEU's.

O terminal Tecon Rio Grande (RS) se mostrou eficiente em alguns períodos, principalmente no início do período em análise. A redução nos escores de eficiência a partir de 2009 pode ser justificada por duas situações observadas: a) estabilidade no número de TEU'S movimentadas nesse período, não acompanhando o aumento na movimentação ocorrido em outros terminais; b) a partir desse ano o terminal passou a operar com um berço a mais e, com a estabilidade na movimentação, pode ter levado à subutilização desse terceiro berço. Dos terminais analisados, apresentou a terceira maior movimentação durante o período. Possui uma capacidade estática para armazenar 39 mil TEU's e uma área de armazenagem de $735 \mathrm{mil} \mathrm{m}^{2}$.

O terminal TECON Santos (SP), referência em modernidade na América do Sul, é o maior e mais eficiente terminal de contêineres do país. Em 2011 atingiu a marca de $80 \mathrm{mph}$ (movimentos por hora), estabelecendo um novo padrão de produtividade na América do Sul, o que o coloca em patamar semelhante aos melhores terminais europeus. Possui 596 mil $\mathrm{m}^{2}$ entre instalações e área de armazenagem, das quais $12 \mathrm{mil} \mathrm{m}^{2}$ são de armazéns cobertos. Possui estrutura para operar navios Super Post Panamax, o que o transformou em um centro de excelência portuária, passando a ser um imenso laboratório de iniciativas inovadoras que visam a melhoria contínua de sua eficiência operacional e rentabilidade. 


\section{Conclusão}

Como forma de observar tendências de crescimento ou redução na produtividade de terminais de contêineres foi aplicado o Modelo de Janelas em conjunto com o modelo BCC orientado para produto, para o período de 2004 a 2011 . Os escores de eficiência gerados mostraram que somente o terminal TECON Santos (SP) mostrou-se eficiente ao longo de todo período analisado. Alguns dos demais terminais se mostraram eficientes em parte do período e outros não alcançaram a fronteira de eficiência no período analisado.

Com base nos dados de movimentação de contêineres, número de acessos e número de berços, pode-se verificar que alguns desses terminais reduziram sua eficiência ou não mostraram crescimento no período, por não conseguirem acompanhar o crescimento geral ocorrido na movimentação de cargas (em TEUs). Além disso, para os terminais TECONDI (SP) e TECON Rio Grande (RS) houve um aumento no número de berços, o que, juntamente com o não aumento na movimentação de contêineres, de acordo com a infraestrutura disponível, pode ter contribuído para a falta de melhorias na produtividade e, consequentemente, na eficiência técnica relativa.

Essa análise pode auxiliar os administradores portuários através da avaliação das causas que podem ter contribuído para o no aumento da movimentação de contêineres em determinado período para cada terminal. De posse dessas informações, as autoridades portuárias podem trabalhar no sentido de captar maior volume de contêineres para o seu terminal, de acordo com sua capacidade, melhorando seu desempenho e se tornando mais competitivos a nível nacional e internacional.

\section{Referências}

Charnes, A. Clark, T., Cooper, W. e Golany, B. (1985). An introduction to Efficiency and Productivity Analysis London: Kluwer Academic Publishers.

Cullinane et. al. (2004). An Application of DEA Windows Analysis to Container Port Production Efficiency. Review of Network Economics, 3 (2), June 2004.

Ferreira, C. M. de C. \& Gomes, A. P. (2009). Introdução à análise envoltória de dados - teoria, modelos e aplicações. Editora UFV Universidade Federal de Viçosa, Viçosa, MG, 389p.

Lovell, K. (1996). Applying Efficiency Measurement Techniques to the Measurement of Productivity Change. Journal of Productivity Analysis, 7 (1), 329-340.

Oliveira, M. S. (2012). Aplicação de uma metodologia para análise de eficiência em terminais de contêineres brasileiros. Dissertação de Mestrado. Programa de Pós-graduação em Engenharia Oceânica, FURG, Rio Grande, RS, 109p.

Oliveira, M. S. ; Azambuja, A. M. V. ; Lima, Milton Luiz Paiva de (2013). Aplicação de uma metodologia para análise de eficiência em terminais de contêineres. Revista Vetor (FURG), v. 23, p. 32-43.

\section{Abstract}

With the large growth of the movement of cargo containers in the last decades, container terminals have been contributing significantly to the supply chain, both, nationally and internationally. As a way to increase productivity, such terminals must know their limitations and potential for the uptake of goods are known. This study aimed to observe the growth trends or reduction in the productivity of Brazilian container terminals, over a given period. The studied period was from 2004 to 2011. For such purpose, Data Envelopment Analysis (DEA) method, also known as windows model, was applied. For windows analysis, estimated score from the BCC model with guidance for product was used. For the mentioned period under review, the terminal Tecon Santos was the only one that remained effective throughout the period. The other terminals, CONVICON (PA), Pound-Rio, Libra Santos (SP) and Tecon Rio Grande (RS), were effective in moving containers during part of the same period.

Key words: container terminals, operational performance, technical efficiency, windows analysis. 